\title{
El ahorro fiscal de las cooperativas en el Impuesto sobre Sociedades en España
}

\author{
Aurelio Herrero Blasco
}

\section{RESUMEN}

El objeto de este artículo es medir el ahorro fiscal que procede del Impuesto sobre Sociedades de las cooperativas en España para el período 1999-2009. La metodología utilizada ha sido un estudio longitudinal en el cual se ha analizado la recaudación total en España por tipos impositivos. Se ha analizado el Impuesto sobre Sociedades en general y posteriormente el Tipo Impositivo Efectivo (TIE) de las Sociedades Cooperativas. Los resultados más importantes son la constatación de diferencias regionales en el pago de los Impuestos sobre Sociedades al aplicarse distintos Tipos Impositivos Efectivos en cada Comunidad Autónoma. Ello supone en la práctica un impacto distinto en la aplicación de las políticas públicas, concretamente en la política fiscal. Se constata, también, una reducida efectividad del impuesto, ya que el ahorro fiscal se concentra sólo en el $43 \%$ de las empresas, que son las que obtienen beneficios.

PALABRAS CLAVE: Tipo impositivo efectivo, ahorro fiscal, políticas públicas, cooperativas, España. CLAVES ECONLIT: D690, H200, H210, H300, H800, H830.

Cómo citar este artículo: HERRERO, A. (2015): "El ahorro fiscal de las cooperativas en el Impuesto sobre Sociedades en España", CIRIEC-España, Revista de Economía Pública, Social y Cooperativa, 84, 279-300.

Correspondencia: Aurelio Herrero Blasco, Profesor Colaborador, Departamento de Organización de Empresas, Universidad Politécnica de Valencia, Edificio 7D. Camino de Vera s/n 46022 Valencia. E-mail: aurelio.herrero@doe.upv.es. 


\title{
Les économies d'impôts des coopératives en matière d'impôt sur les sociétés en Espagne
}

RÉSUMÉ : Le but de cet article est de mesurer les économies d'impôts résultant de l'impôt sur les sociétés des coopératives en Espagne entre 1999 et 2009. Nous avons utilisé une méthodologie d'étude longitudinale dans le cadre de laquelle le recouvrement total en Espagne a été analysé en fonction des taux d'imposition. Nous avons notamment examiné l'impôt sur les sociétés dans son ensemble, puis avons analysé le taux d'imposition effectif (TIE) des sociétés coopératives. L'un des principaux résultats de cet article est la constatation de différences régionales dans le paiement des impôts sur les sociétés, en raison de l'application de différents taux d'imposition effectifs dans chaque Communauté autonome. Cela implique, au niveau pratique, un impact distinct sur la mise en œuvre des politiques publiques, en particulier de la politique fiscale. On observe également une baisse de l'efficacité de l'impôt, car les économies d'impôts sont concentrées sur seulement 43 \% des entreprises, qui sont celles réalisant des profits.

MOTS CLÉ : Taux d'imposition effectif, économies d'impôt, politiques publiques, coopératives, Espagne.

\section{Corporate Income Tax savings in Spanish cooperatives}

\begin{abstract}
This study aimed to measure the savings in corporate income tax made by cooperatives in Spain during the 1999-2009 period. The method used was a longitudinal study that analysed the total revenue collected in Spain by tax rate, first examining corporate income tax in general and then the effective tax rate (ETR) paid by cooperative societies. The most important finding is the existence of regional differences in corporate income tax payments, as different regions apply different effective tax rates. In practice, this means that there are variations in the impact of public policies, specifically tax policies. It was also found that the effectiveness of this tax is limited, as the tax savings were concentrated in only $43 \%$ of companies, which are those that make a profit.
\end{abstract}

KEY WORDS: Effective tax rate, tax savings, public policy, cooperatives, Spain. 


\section{EL AHORRO FISCAL DE LAS COOPERATIVAS EN EL IMPUESTO SOBRE SOCIEDADES EN ESPAÑA}

\section{1.- Introducción}

Las principales motivaciones de este trabajo son dos: la de reflexionar acerca del diferente impacto territorial que produce la política pública de incentivo fiscal de las cooperativas y, también, destacar la reducida efectividad de dicho incentivo, al afectar sólo al 43,24\% de media de éstas, que son las que obtienen beneficios y, por tanto, pueden aplicar a su cuenta de resultados un tipo de gravamen más ventajoso que el que soportan las sociedades capitalistas.

El objetivo principal de este trabajo es el de cuantificar el ahorro fiscal de las cooperativas en España, ahorro que procede de la ventaja fiscal que tienen éstas respecto a las sociedades capitalistas en el Impuesto sobre Sociedades, debido al incentivo fiscal que tienen dichas sociedades cooperativas en el tipo de gravamen que se aplica a la base imponible que corresponde a los resultados cooperativos. Al cuantificar este ahorro fiscal se conoce el impacto de la política pública de incentivo fiscal en las cooperativas a través del Impuesto sobre Sociedades.

En concreto se ha realizado dicho estudio para el período comprendido entre los años 1999 y 2009. Se justifica este período temporal porque fundamentalmente se ha trabajado con la base de datos del Ministerio de Trabajo (ahora Ministerio de Empleo y de la Seguridad Social) y esta base de datos tiene su inicio en el año 1999 y el año final 2009, último año en que había datos disponibles a mediados del año 2012, fecha de cierre del estudio.

La metodología utilizada es un estudio de panel para dicho período y mediante este estudio longitudinal, primero se ha analizado la recaudación total en España por tipos impositivos para ver el peso y la importancia de cada uno de ellos, en particular del Impuesto sobre Sociedades, que es el objeto de este trabajo, para en una fase posterior analizar cuál es el Tipo Impositivo Efectivo (TIE) de las Sociedades Cooperativas.

Posteriormente se ha calculado la ventaja fiscal o ahorro fiscal calculando la diferencia de Tipo Impositivo Efectivo ( $\mathrm{TIE}$ ) que tienen las Cooperativas respecto a las Sociedades Laborales, que en este estudio asimilamos a las empresas que no tienen ventajas fiscales y por lo tanto nos sirve como grupo de control y se han equiparado con las empresas capitalistas que tributan a los mismos tipos impositivos que las Sociedades Laborales.

El cálculo del Tipo Impositivo Efectivo (TIE) se hace dividiendo el importe del Impuesto sobre Beneficios realmente recaudado sobre el Beneficio antes de Impuestos (BAI), declarado en las Cuentas de Pérdidas y Ganancias de las empresas estudiadas. 
El estudio se basa, fundamentalmente, en las series estadísticas proporcionadas por la Agencia Estatal de la Administración Tributaria, del Ministerio de Economía y Hacienda a través de la Subdirección General de Política Tributaria y del Ministerio de Trabajo y Empleo, concretamente de la Dirección General de Cooperativas. En estas estadísticas la acepción Economía Social incluye las Cooperativas, las Sociedades Anónimas Laborales y las Sociedades Limitadas Laborales.

Se ha tomado el año 2006 como año de referencia y control por un doble motivo: en primer lugar, porque está en la mitad de la serie de once años y, en segundo lugar, porque casualmente la serie en el año 2007 empieza a notar los efectos de la crisis económica actual.

Se calcula la Ventaja o Ahorro Fiscal de la siguiente manera: (TIE Sociedades Laborales en porcentaje menos la TIE Sociedades Cooperativas en porcentaje) multiplicado por lo recaudado en concepto de Impuesto sobre Sociedades de las Sociedades Cooperativas. De tal manera se calcula lo dejado de recaudar en términos monetarios comparado con lo que han pagado las Sociedades Laborales, en dicho periodo, por dicho concepto.

Para establecer el estado del arte se ha recurrido al estudio de los diferentes autores especialistas en la materia tanto en el ámbito fiscal, de economía social, como en lo referente a políticas públicas [Albi, González-Páramo y Zubiri (2009), Alguacil (2011), Calvo (2005), Chaves (2012), Chaves y Monzón (2008), Juliá y Server (1996), Marín y Server (2004), Monserrat (1991), Rodrigo (2010), Fernández, Martínez y Álvarez (2008), Domínguez. López y Rodrigo (2005)]. Las bases de datos utilizadas han sido las de la Agencia Tributaria, Ministerio de Empleo y Seguridad Social, Ministerio de Hacienda y Administraciones Públicas y Observatorio Español de la Economía Social.

El presente artículo se estructura de la siguiente manera: en primer lugar se analiza el sistema recaudatorio en España para averiguar la presión fiscal sobre el Producto Interior Bruto y la magnitud o importancia de la recaudación directa e indirecta, con el objetivo de estimar la capacidad recaudatoria del Impuesto sobre Sociedades, todo ello comparado con la Unión Europea a 27 miembros, y con el fin de conocer la posición de España respecto al resto de sus socios europeos. En segundo lugar, se estudia cuál es la aportación que realiza la economía social, en general, y las sociedades cooperativas, en particular, a dicho Impuesto sobre Sociedades. En tercer lugar, se calcula el Tipo Impositivo Efectivo de la economía social y de las cooperativas, en particular, y se calcula el ahorro o ventaja fiscal. Para finalizar, se compara este ahorro fiscal por Comunidades Autónomas y por clases de cooperativas y se extraen las conclusiones. 


\section{2.- Estructura recaudatoria en Españar impuestos directos, impuestos indirectos, tasas y otros impuestos}

En este punto se analizan las magnitudes de la recaudación general por los capítulos I, Impuestos Directos; II, Impuestos Indirectos, y III, Tasas y otros impuestos en España desde 1999 a 2009.

Esta estructura recaudatoria está constituida por Capítulo I, Impuestos directos, que son:

- Impuesto sobre la Renta de las personas Físicas el más cuantioso en términos brutos y en porcentaje.

- Impuesto sobre la Renta de no Residentes, de carácter complementario.

- Impuesto sobre Sociedades, el segundo más cuantioso y objeto de la investigación.

- Impuesto sobre Sucesiones y Donaciones e Impuesto sobre el Patrimonio, cedidos a las Comunidades Autónomas, de menor importancia.

Capítulo II, Impuestos indirectos, que son:

- Impuesto sobre el Valor Añadido, de poder recaudatorio similar al de la renta de las personas físicas.

- Impuestos Especiales, fundamentalmente recaen sobre el Alcohol, Tabaco e Hidrocarburos, también de gran importancia.

- Impuesto sobre Transmisiones Patrimoniales y Actos Jurídicos Documentados, cedidos a las Comunidades Autónomas.

- Gravamen sobre el Tráfico Exterior de Mercancías, Cotización y Exacción de Azúcar e Isoglucosa e Impuesto sobre primas de seguros, de menor importancia.

Capítulo III, Tasas y otros ingresos, que son:

- Tasas sobre el juego, Tasa dominio público radioeléctrico, Otras tasas, Recargo de apremio, intereses de demora, Sanciones tributarias, otras. 
Tabla 1. Recaudación en España. Capítulos I, II y III. Millones de euros. Periodo 1999-2009

\begin{tabular}{|c|c|c|c|c|c|c|}
\hline & 1999 & 2000 & 2001 & 2002 & 2003 & 2004 \\
\hline Impuesto sobre la Renta de las Personas Físicas & 30.707 & 32.119 & 36.127 & 32.276 & 32.829 & 30.412 \\
\hline Impuesto sobre Sociedades & 14.635 & 17.206, & 17.215, & 21.437 & 21.919 & 26.019, \\
\hline Impuesto sobre la Renta de no Residentes & 723 & 1.098 & 905,7 & $1.051,6$ & 947,8 & $1.395,4$ \\
\hline Impuesto sobre Sucesiones y Donaciones & 12,0 & 9 & 10,6 & 15,9 & 16,2 & 13,7 \\
\hline Impuesto sobre el Patrimonio & 271,2 & 324 & 338,4 & 27,6 & 31,4 & 30,4 \\
\hline Resto Capítulo I & 0,9 & -2 & 0,5 & $-2,6$ & 1,1 & 5,0 \\
\hline \multirow[t]{2}{*}{ Total Capítulo I: impuestos directos } & 46.350 & 50.756 & 54.597, & 54.806 & 55.745 & 57.876, \\
\hline & 1999 & 2000 & 2001 & 2002 & 2003 & 2004 \\
\hline Impuesto sobre el Valor Añadido & 30.734 & 33.389, & 34.672, & 25.728 & 27.140 & 29.123 , \\
\hline Impuestos Especiales & 15.425 & 16.056 & 16.576 & 10.382 & 9.786 & 9.751 \\
\hline Impuesto sobre Transmisiones Patrimoniales y Actos & & & & & & \\
\hline Jurídicos Documentados & 10,5 & 9,6 & 9,0 & 9,9 & 6,9 & 7,4 \\
\hline Gravamen sobre el Tráfico Exterior de Mercancías & 867,0 & 969,2 & 952,8 & 923,1 & 1.020 & $1.232,0$ \\
\hline Cotización y Exacción de Azúcar e Isoglucosa & 48,0 & 48,9 & 33,6 & 23,0 & 18,0 & 20,3 \\
\hline Impuesto sobre las Primas de Seguros & 688,2 & 789,2 & 878,5 & 1.003, & 1.125 & $1.233,2$ \\
\hline Resto Capítulo II & 9,1 & 7,6 & $-3,0$ & 1,2 & $-2,1$ & 0,3 \\
\hline \multirow[t]{2}{*}{ Total Capítulo II: impuestos indirectos } & 47.782 & 51.270, & 53.119, & 38.072 & 39.096 & 41.367, \\
\hline & 1999 & 2000 & 2001 & 2002 & 2003 & 2004 \\
\hline Tasas sobre el juego & 270,1 & 291,0 & 297,3 & 12,3 & 9,7 & 13,0 \\
\hline Tasa dominio público radioeléctrico & .. & .. & 457,4 & 454,0 & 245,3 & 226,5 \\
\hline Otras tasas & 333,9 & 360,4 & 321,1 & 300,3 & 380,2 & 399,1 \\
\hline Recargo de apremio & 136,7 & 141,2 & 143,3 & 155,2 & 176,7 & 186,4 \\
\hline Intereses de demora & 205,5 & 160,2 & 176,0 & 152,2 & 190,8 & 144,2 \\
\hline Sanciones tributarias & 80,3 & 89,9 & 106,4 & 110,5 & 124,8 & 109,1 \\
\hline Otros ingresos & 56,5 & 48,5 & 88,9 & 61,6 & 80,7 & 74,5 \\
\hline \multirow[t]{2}{*}{ Total Capítulo III: tasas y otros ingresos } & 1.083, & $1.091,1$ & $1.590,4$ & 1.246, & 1.208, & $1.152,8$ \\
\hline & 1999 & 2000 & 2001 & 2002 & 2003 & 2004 \\
\hline Total Capítulos I, II y III & 95.215 & 103.117 & 109.307 & 94.125 & 96.049 & 100.397 \\
\hline
\end{tabular}

FUENTE: Dirección General de Tributos. S.G. Política Tributaria, 2012. 


\section{Tabla 2. Recaudación en España. Capítulos I, II y III. Millones de euros. Periodo 2005-2009}

\begin{tabular}{|c|c|c|c|c|c|}
\hline & 2005 & 2006 & 2007 & 2008 & 2009 \\
\hline Impuesto sobre la Renta de las Personas Físicas & 35.952 & 41.495, & 48.625, & 43.413, & 30.432 \\
\hline Impuesto sobre Sociedades & 32.495, & 37.207, & 44.823, & 27.301, & 20.188, \\
\hline Impuesto sobre la Renta de no Residentes & 1.350 & 1.498, & 2.427, & 2.262, & 2.341, \\
\hline Impuesto sobre Sucesiones y Donaciones & 24 & 34 & 66 & 54 & 68 \\
\hline Impuesto sobre el Patrimonio & 37,8 & 40,9 & 47,2 & 52,2 & 6,2 \\
\hline Resto Capítulo I & 1,0 & 1,3 & 1,0 & $-0,2$ & $-0,5$ \\
\hline \multirow[t]{2}{*}{ Total Capítulo I: impuestos directos } & 69.861, & 80.277, & 95.990 & 73.083 & 53.036, \\
\hline & 2005 & 2006 & 2007 & 2008 & 2009 \\
\hline Impuesto sobre el Valor Añadido & 32.009 & 35.423 & 33.752 & 24.928, & 15.777 \\
\hline Impuestos Especiales & $9.795,0$ & $9.894,8$ & $11.467,8$ & $11.220,0$ & $10.140,7$ \\
\hline Impuesto sobre Transm. Patrimoniales y Actos Jurídicos Documentados & 9,6 & 11,4 & 12,4 & 8,0 & 5,9 \\
\hline Gravamen sobre el Tráfico Exterior de Mercancías & $1.458,5$ & $1.595,4$ & $1.720,4$ & $1.566,1$ & $1.318,5$ \\
\hline Cotización y Exacción de Azúcar e Isoglucosa & 27,0 & 7,9 & $-1,7$ & 8,2 & 7,8 \\
\hline Impuesto sobre las Primas de Seguros & $1.314,0$ & $1.387,1$ & $1.491,3$ & $1.501,7$ & $1.406,1$ \\
\hline Resto Capítulo II & 4,0 & 5,6 & 1,9 & 1,8 & 1,0 \\
\hline \multirow[t]{2}{*}{ Total Capítulo II: impuestos indirectos } & 44.617, & 48.326, & 48.444, & 39.234, & 28.657, \\
\hline & 2005 & 2006 & 2007 & 2008 & 2009 \\
\hline Tasas sobre el juego & 15,3 & 21,0 & 24,6 & 18,3 & 18,8 \\
\hline Tasa dominio público radioeléctrico & 243,1 & 351,0 & 524,6 & 321,9 & 315,7 \\
\hline Otras tasas & 529,3 & 502,0 & 597,2 & 681,7 & 728,0 \\
\hline Recargo de apremio & 177,0 & 192,2 & 216,1 & 237,0 & 289,0 \\
\hline Intereses de demora & 169,2 & 160,1 & 169,1 & 173,6 & 221,2 \\
\hline Sanciones tributarias & 168,5 & 197,2 & 210,4 & 207,3 & 194,3 \\
\hline Otros ingresos & 65,5 & 99,0 & 93,6 & 125,5 & 140,2 \\
\hline \multirow[t]{2}{*}{ Total Capítulo III: tasas y otros ingresos } & $1.367,9$ & $1.522,6$ & $1.835,6$ & $1.765,3$ & $1.907,2$ \\
\hline & 2005 & 2006 & 2007 & 2008 & 2009 \\
\hline Total Capítulos I, II y III & 115.846 & 130.126 & 146.270 & 114.082 & 83.601, \\
\hline
\end{tabular}

FUENTE: Dirección General de Tributos. S.G. Política Tributaria, 2012. 
La recaudación por Impuesto sobre Sociedades total en España supuso en 1999 la cantidad de 14.635,6 millones de euros, es decir el 15,37\% de la recaudación total de los capítulos I, II y III (Impuestos directos 46.350,1 millones de euros, el 48,68\% del total + Impuestos Indirectos 47.782,4 millones de euros, el 50,18\% del total + Tasas y otros ingresos 1.083,0 millones de euros, el 1,14\% del total). El total recaudado es 95.215 .5 millones de euros. El IRPF ese año es $30.707,5$ millones de euros y el IVA 30.734,6 millones de euros, ambos superan claramente al Impuesto sobre Sociedades (IS).

\section{Tabla 3. Magnitud de los impuestos IRPF, IVA y Sociedades en el conjunto de la recaudación en España, \%}

\begin{tabular}{|l|rrrrrc|}
\hline & $\mathbf{1 9 9 9}$ & $\mathbf{2 0 0 0}$ & $\mathbf{2 0 0 1}$ & $\mathbf{2 0 0 2}$ & $\mathbf{2 0 0 3}$ & $\mathbf{2 0 0 4}$ \\
\hline IRPF & 32,25 & 31,15 & 33,05 & 34,29 & 34,18 & 30,29 \\
IVA & 32,28 & 32,38 & 31,72 & 27,33 & 28,26 & 29,01 \\
SOCIEDADES & 15,37 & 16,69 & 15,75 & 22,78 & 22,82 & 25,92 \\
\hline & $\mathbf{2 0 0 5}$ & $\mathbf{2 0 0 6}$ & $\mathbf{2 0 0 7}$ & $\mathbf{2 0 0 8}$ & $\mathbf{2 0 0 9}$ & PROMEDIO \\
\hline IRPF & 31,03 & 31,89 & 33,24 & 38,05 & 36,4 & 33,26 \\
IVA & 27,63 & 27,22 & 23,08 & 21,85 & 18,87 & 27,24 \\
SOCIEDADES & 28,05 & 28,59 & 30,64 & 23,93 & 24,15 & 23,15 \\
\hline
\end{tabular}

FUENTE: Elaboración propia en base a Dirección General de Tributos. S.G. Política Tributaria, 2012.

La recaudación por Impuesto sobre sociedades representó sólo el 15,37\% de la recaudación total por impuestos en España en 1999. Esta cantidad pasó a ser en 2009 del 24,14\%.

\section{3.- Presión fiscal en España en relación al Producto Interior Bruto}

La presión fiscal en España, entendida como proporción entre ingresos fiscales totales (tributos más cotizaciones sociales) y PIB ha sido para 1998 del 33,0\% y ha ido evolucionando alrededor de esa magnitud hasta el 2008. La media de la Unión Europea 27 miembros ha sido del 40,3\% en 1998. Según los datos ofrecidos la principal fuente de ingreso son las Cotizaciones Sociales que eran de un $11,9 \%$ en 1998 y un $12,3 \%$ en 2008 sobre el PIB. 
Tabla 4. Porcentaje en que contribuye cada impuesto a la totalidad de la recaudación en España 1998-2008

\begin{tabular}{|c|c|c|c|c|c|c|}
\hline & 1998 & 1999 & 2000 & 2001 & 2002 & 2003 \\
\hline ID. IRPF & $7,00 \%$ & $6,70 \%$ & $6,60 \%$ & $6,80 \%$ & $6,80 \%$ & $6,60 \%$ \\
\hline ID.SOCIEDADES & $2,50 \%$ & $2,90 \%$ & $3,10 \%$ & $2,90 \%$ & $3,30 \%$ & $3,10 \%$ \\
\hline ID.OTROS & $0,70 \%$ & $0,80 \%$ & $0,80 \%$ & $0,80 \%$ & $0,70 \%$ & $0,80 \%$ \\
\hline COTIZACIONES SOCIALES & $11,90 \%$ & $11,90 \%$ & $12,00 \%$ & $12,20 \%$ & $12,10 \%$ & $12,20 \%$ \\
\hline II. IVA & $5,60 \%$ & $6,10 \%$ & $6,10 \%$ & $5,90 \%$ & $5,80 \%$ & $6,00 \%$ \\
\hline II.ESPECIALES & $2,80 \%$ & $2,70 \%$ & $2,60 \%$ & $2,50 \%$ & $2,50 \%$ & $2,50 \%$ \\
\hline II.OTROS & $3,10 \%$ & $3,20 \%$ & $3,20 \%$ & $3,10 \%$ & $3,20 \%$ & $3,40 \%$ \\
\hline \multirow[t]{2}{*}{ SUMA } & $33,60 \%$ & $34,30 \%$ & $34,40 \%$ & $34,20 \%$ & $34,40 \%$ & $34,60 \%$ \\
\hline & 2004 & 2005 & 2006 & 2007 & 2008 & PROMEDIO \\
\hline ID. IRPF & $6,40 \%$ & $6,60 \%$ & $7,10 \%$ & $7,70 \%$ & $7,50 \%$ & $6,89 \%$ \\
\hline ID.SOCIEDADES & $3,50 \%$ & $3,90 \%$ & $4,20 \%$ & $4,80 \%$ & $2,90 \%$ & $3,37 \%$ \\
\hline ID.OTROS & $0,80 \%$ & $0,80 \%$ & $0,90 \%$ & $0,90 \%$ & $0,90 \%$ & $0,81 \%$ \\
\hline COTIZACIONES SOCIALES & $12,20 \%$ & $12,10 \%$ & $12,10 \%$ & $12,20 \%$ & $12,30 \%$ & $12,11 \%$ \\
\hline II. IVA & $6,10 \%$ & $6,30 \%$ & $6,40 \%$ & $6,10 \%$ & $5,30 \%$ & $5,97 \%$ \\
\hline II.ESPECIALES & $2,50 \%$ & $2,40 \%$ & $2,20 \%$ & $2,20 \%$ & $2,10 \%$ & $2,45 \%$ \\
\hline II.OTROS & $3,60 \%$ & $3,90 \%$ & $4,10 \%$ & $3,70 \%$ & $2,80 \%$ & $3,39 \%$ \\
\hline SUMA & $35,10 \%$ & $36,00 \%$ & $37,00 \%$ & $37,60 \%$ & $33,80 \%$ & $35,00 \%$ \\
\hline
\end{tabular}

FUENTE: Elaboración propia en base a Dirección General de Tributos. S.G. política Tributaria, 2012.

\section{4.- Presión fiscal en España comparada con la media de la Unión Europea a 27 miembros}

En España, en 1998 el Impuesto sobre Sociedades representaba el 2,5\% del PIB, mientras que la recaudación por Impuesto sobre la Renta de las Personas Físicas (IRPF) era del 7,0\% sobre el PIB, las cotizaciones sociales el 11,9\% y la recaudación por Impuesto sobre el Valor Añadido del 5,6\% (Comisión Europea). Todo esto hace reflexionar acerca del poder recaudatorio del Impuesto sobre Sociedades. 
Tabla 5. Presión fiscal sobre el PIB. Países de Europa UE. 27, 2003-2007

\begin{tabular}{|c|c|c|c|c|c|}
\hline País & 2003 & 2004 & 2005 & 2006 & 2007 \\
\hline Alemania & $39,6 \%$ & $38,7 \%$ & $38,8 \%$ & $39,2 \%$ & $39,4 \%$ \\
\hline Austria & $43,8 \%$ & $43,4 \%$ & $42,3 \%$ & $41,9 \%$ & $42,2 \%$ \\
\hline Bélgica & $44,6 \%$ & $44,7 \%$ & $44,7 \%$ & $44,3 \%$ & $43,9 \%$ \\
\hline Bulgaria & $32,2 \%$ & $33,1 \%$ & $34,0 \%$ & $33,2 \%$ & $34,2 \%$ \\
\hline Chipre & $33,0 \%$ & $33,4 \%$ & $35,5 \%$ & $36,5 \%$ & $40,9 \%$ \\
\hline Dinamarca & $48,0 \%$ & $49,0 \%$ & $50,8 \%$ & $49,6 \%$ & $49,0 \%$ \\
\hline Eslovenia & $38,2 \%$ & $38,3 \%$ & $38,6 \%$ & $38,3 \%$ & $37,8 \%$ \\
\hline España & $33,9 \%$ & $34,5 \%$ & $35,6 \%$ & $36,4 \%$ & $37,1 \%$ \\
\hline Estonia & $30,8 \%$ & $30,6 \%$ & $30,6 \%$ & $31,1 \%$ & $32,3 \%$ \\
\hline Finlandia & $44,0 \%$ & $43,5 \%$ & $44,0 \%$ & $43,5 \%$ & $43,0 \%$ \\
\hline Francia & $42,9 \%$ & $43,2 \%$ & $43,6 \%$ & $43,9 \%$ & $43,2 \%$ \\
\hline Grecia & $32,1 \%$ & $31,2 \%$ & $31,8 \%$ & $31,7 \%$ & $32,4 \%$ \\
\hline Holanda & $37,4 \%$ & $37,5 \%$ & $37,6 \%$ & $39,0 \%$ & $38,9 \%$ \\
\hline Hungría & $37,9 \%$ & $37,4 \%$ & $37,5 \%$ & $37,2 \%$ & $39,8 \%$ \\
\hline Irlanda & $29,0 \%$ & $30,3 \%$ & $30,8 \%$ & $32,3 \%$ & $31,4 \%$ \\
\hline Italia & $41,3 \%$ & $40,6 \%$ & $40,4 \%$ & $42,0 \%$ & $43,1 \%$ \\
\hline Letonia & $28,5 \%$ & $28,5 \%$ & $29,0 \%$ & $30,4 \%$ & $30,5 \%$ \\
\hline Lituania & $28,1 \%$ & $28,3 \%$ & $28,5 \%$ & $29,4 \%$ & $29,7 \%$ \\
\hline Luxemburgo & $38,1 \%$ & $37,3 \%$ & $37,6 \%$ & $35,6 \%$ & $35,7 \%$ \\
\hline Malta & $31,4 \%$ & $32,9 \%$ & $33,9 \%$ & $33,7 \%$ & $34,6 \%$ \\
\hline Polonia & $32,2 \%$ & $31,5 \%$ & $32,8 \%$ & $33,8 \%$ & $34,8 \%$ \\
\hline Portugal & $34,8 \%$ & $34,1 \%$ & $35,1 \%$ & $35,9 \%$ & $36,8 \%$ \\
\hline Reino Unido & $34,7 \%$ & $35,1 \%$ & $36,0 \%$ & $36,8 \%$ & $36,5 \%$ \\
\hline República Checa & $35,7 \%$ & $37,4 \%$ & $37,1 \%$ & $36,7 \%$ & $37,2 \%$ \\
\hline República Eslovaca & $32,9 \%$ & $31,5 \%$ & $31,3 \%$ & $29,2 \%$ & $29,3 \%$ \\
\hline Rumanía & $27,7 \%$ & $27,2 \%$ & $27,8 \%$ & $28,5 \%$ & $29,0 \%$ \\
\hline Suecia & $48,3 \%$ & $48,7 \%$ & $49,5 \%$ & $49,0 \%$ & $48,3 \%$ \\
\hline Unión Europea-16 $\left(^{\star}\right)$ & $39,8 \%$ & $39,5 \%$ & $39,6 \%$ & $40,2 \%$ & $40,4 \%$ \\
\hline Unión Europea-25 $\left(^{*}\right)$ & $39,1 \%$ & $38,9 \%$ & $39,3 \%$ & $39,8 \%$ & $39,9 \%$ \\
\hline Unión Europea-27 ${ }^{*}$ ) & $39,0 \%$ & $38,9 \%$ & $39,2 \%$ & $39,7 \%$ & $39,7 \%$ \\
\hline
\end{tabular}

$\left({ }^{*}\right)$ Medias ponderadas

Presión Fiscal = tributos más cotización a la Seguridad Social.

FUENTE: Elaboración propia en base a Dirección General de Tributos. S.G. Política Tributaria, 2012.

Como se puede observar en la Tabla 5 para el año 2006, por ejemplo, España tenía una presión fiscal sobre el PIB del $36,4 \%$, Alemania del $39,2 \%$ y la presión fiscal media de la UE-27 era del $39,7 \%$. 


\section{5.- Aportación de las cooperativas y sociedades laborales a la recaudación del Impuesto sobre Sociedades en España}

De la recaudación por Impuesto sobre Sociedades del año 1999, que asciende a 14.635 .6 millones de euros, las cooperativas y sociedades laborales aportan 68,08 millones de euros, que suponen el $0,46 \%$ del total. Las Sociedades Cooperativas aportan al Impuesto sobre Sociedades 36,59 millones de euros, que suponen el $0,25 \%$ del total.

Esta situación aparecía en el año 2009 de la siguiente manera: de la recaudación por Impuesto sobre Sociedades del año 2009 (20.188,5 millones de euros), la Economía Social aportaba 41,91 millones de euros, que suponen el $0,21 \%$ del total.

\section{Tabla 6. Contribución de las Cooperativas y Sociedades Laborales al total de recaudación por Impuesto sobre Sociedades en España 1999-2004. Millones de euros y \%}

\begin{tabular}{|l|rrrrrr|}
\hline & $\mathbf{1 9 9 9}$ & $\mathbf{2 0 0 0}$ & $\mathbf{2 0 0 1}$ & $\mathbf{2 0 0 2}$ & $\mathbf{2 0 0 3}$ & $\mathbf{2 0 0 4}$ \\
\hline Cooperativas & 36,60 & 36,92 & 31,75 & 52,18 & 52,29 & 54,07 \\
Soc. Laborales & 31,49 & 43,86 & 41,43 & 48,43 & 62,03 & $\mathbf{7 6 , 1 3}$ \\
Economía Social & 68,09 & 80,78 & 73,18 & 100,61 & 114,32 & 130,20 \\
Total España I. Sociedades & $14.635,60$ & $17.206,60$ & $17.215,20$ & $21.437,00$ & $21.919,60$ & $26.019,90$ \\
\% Cooperativa & $0,25 \%$ & $0,22 \%$ & $0,18 \%$ & $0,24 \%$ & $0,24 \%$ & $0,21 \%$ \\
\% S. Lab. & $0,21 \%$ & $0,25 \%$ & $0,24 \%$ & $0,23 \%$ & $0,28 \%$ & $0,29 \%$ \\
\% ES & $0,46 \%$ & $0,47 \%$ & $0,43 \%$ & $0,47 \%$ & $0,52 \%$ & $0,50 \%$ \\
\hline
\end{tabular}

FUENTE: Elaboración propia en base a Dirección General de Tributos. S.G. Política Tributaria, 2012. 


\section{Tabla 7. Contribución de las Cooperativas y Sociedades Laborales al total de recaudación por Impuesto sobre Sociedades en España 2005-2009}

\begin{tabular}{|l|rrrrrr|}
\hline Año & $\mathbf{2 0 0 5}$ & $\mathbf{2 0 0 6}$ & $\mathbf{2 0 0 7}$ & $\mathbf{2 0 0 8}$ & $\mathbf{2 0 0 9}$ & PROMEDIO \\
\hline Cooperativas & 73,87 & 74,94 & 33,72 & 6,27 & 18,30 & 42,81 \\
Soc. Laborales & 110,09 & 133,21 & 123,28 & 54,61 & 23,62 & 68,02 \\
Economía Social & 183,96 & 208,15 & 157,00 & 60,88 & 41,92 & 110,83 \\
Total España I. Sociedades & $32.495,30$ & $37.207,60$ & $44.823,20$ & $27.301,40$ & $20.188,50$ & $25.495,45$ \\
\% Cooperativa & 0,23 & 0,20 & 0,08 & 0,02 & 0,10 & 0,18 \\
\% S. Lab. & 0,34 & 0,36 & 0,28 & 0,20 & 0,11 & 0,25 \\
\% ES & 0,57 & 0,56 & 0,35 & 0,22 & 0,21 & 0,43 \\
\hline
\end{tabular}

FUENTE: Elaboración propia en base a Dirección General de Tributos. S.G. Política Tributaria, 2012.

En el año 2006, en España por Impuesto sobre Sociedades se recaudó 37.207,60 millones de euros. La recaudación de la Economía Social (Cooperativas + Sociedades Laborales) fue de 208,15 millones de euros, que representan el $0,56 \%$ del total.

\section{6.- El Tipo Impositivo Efectivo en la recaudación por impuesto sobre sociedades de la Economía Social en general y de las Cooperativas en particular}

El tipo impositivo efectivo (TIE) representa el cociente entre lo recaudado efectivamente y los Beneficios Antes de Impuestos presentados a la Agencia Estatal de la Administración Tributaria. Se estiman los TIE para las Sociedades Cooperativas, para las Sociedades Laborales y conjuntamente.

El cálculo de la Ventaja Fiscal se basa en la analogía de que la mayoría de las pequeñas y medianas empresas tributarían a tipos similares a los de las Sociedades Laborales. Así, el cálculo, por ejemplo, para el año 1999, si las Cooperativas pagaron Impuesto sobre Sociedades por importe de 36.595.60 miles de euros con un Tipo Impositivo Efectivo (TIE) del 7,59\%, y deberían haber pagado como las Sociedades laborales a un $20,76 \%$, entonces han obtenido una Ventaja Fiscal porcentual de $13,17 \%$, y en valores absolutos de 63.499 .88 miles de euros. Desde 1999 a 2009 se estima que las Cooperativas han obtenido un Ahorro Fiscal de 1.002.284,65 miles de euros sobre las Sociedades Laborales. 


\section{Tabla 8. Tipos Impositivos Efectivos de Cooperativas y Sociedades Laborales, 1999-2009}

\begin{tabular}{|cccc|}
\hline AÑO & \% TIE E. SOCIAL & \% TIE COOPERATIVAS & \% TIE S. LABORALES \\
\hline 1999 & 10,75 & 7,59 & 20,76 \\
2000 & 11,80 & 7,35 & 24,03 \\
2001 & 9,48 & 5,63 & 19,91 \\
2002 & 11,99 & 8,94 & 18,96 \\
2003 & 11,00 & 7,05 & 20,84 \\
2004 & 12,76 & 8,28 & 18,19 \\
2005 & 13,87 & 8,62 & 23,45 \\
2006 & 13,64 & 7,85 & 23,33 \\
2007 & 9,99 & 3,48 & 20,47 \\
2008 & 5,04 & 0,80 & 12,80 \\
2009 & 5,32 & 3,67 & 8,16 \\
\hline
\end{tabular}

FUENTE: Elaboración propia en base a Dirección General de Tributos. S.G. política Tributaria, 2012.

\section{Tabla 9. Recaudación y ahorro fiscal de las Cooperativas y Sociedades Laborales, 1999-2009. Miles de euros}

\begin{tabular}{|c|c|c|c|c|}
\hline AÑO & $\begin{array}{r}\text { Recaudación } \\
\text { Economía } \\
\text { Social }\end{array}$ & $\begin{array}{l}\text { Recaudación } \\
\text { Cooperativas }\end{array}$ & $\begin{array}{r}\text { Recaudación } \\
\text { Sociedades } \\
\text { laborales }\end{array}$ & $\begin{array}{r}\text { Ahorro } \\
\text { fiscal de las } \\
\text { cooperativas }\end{array}$ \\
\hline 1999 & $68.087,78$ & $36.595,60$ & $31.492,18$ & 63.499 \\
\hline 2000 & $80.776,29$ & $36.920,64$ & $43.855,65$ & 83.787 \\
\hline 2001 & $73.178,54$ & $31.745,19$ & $41.433,35$ & 80.518 \\
\hline 2002 & $100.614,56$ & $52.177,40$ & $48.437,16$ & 58.480 \\
\hline 2003 & $114.317,18$ & $52.289,23$ & $62.027,95$ & 102.279 \\
\hline 2004 & $130.201,15$ & $54.068,41$ & $72.930,79$ & 57.948 \\
\hline 2005 & $183.955,33$ & $73.780,77$ & $110.084,56$ & 126.933 \\
\hline 2006 & $208.148,00$ & $74.942,77$ & $133.205,23$ & 147.785 \\
\hline 2007 & $157.004,85$ & $33.718,89$ & $123.285,96$ & 164.621 \\
\hline 2008 & $60.878,39$ & $6.269,58$ & $54.608,81$ & 94.043 \\
\hline 2009 & $41.915,63$ & $18.297,84$ & $23.617,79$ & 22.386 \\
\hline SUMA & $1.219 .077,7$ & $470.806,32$ & $744.979,43$ & 1.002 .284 \\
\hline & & & PROMEDIO & 91.116 \\
\hline
\end{tabular}

FUENTE: Elaboración propia en base a Dirección General de Tributos. S.G. Política Tributaria, 2012. 
Como se puede comprobar, el tipo efectivo del Impuesto sobre Sociedades en la Economía Social evoluciona desde un 10,75\% en el año 1999 hasta un 5,32\% en 2009. Las Cooperativas evolucionan desde un tipo efectivo impositivo del Impuesto sobre Sociedades del 7,59\% en el año 1999 al $3,67 \%$ en 2009. El resto lo explican las Sociedades Laborales (anónimas y limitadas).

\section{Tabla 10. Comparación entre los Tipos de Imposición Efectivo del Impuesto Sobre Sociedades, 2003-2009}

\begin{tabular}{|lrrrrrrr|}
\hline & $\mathbf{2 0 0 3}$ & $\mathbf{2 0 0 4}$ & $\mathbf{2 0 0 5}$ & $\mathbf{2 0 0 6}$ & $\mathbf{2 0 0 7}$ & $\mathbf{2 0 0 8}$ & $\mathbf{2 0 0 9}$ \\
\hline Tipo efectivo Total & 24,30 & 25,00 & 24,5 & 23,30 & 19,90 & 18,00 & 16,70 \\
Tipo efectivo Cooperativas & 7,05 & 8,78 & 8,62 & 7,85 & 3,48 & 0,80 & 3,67 \\
Tipo efectivo S. Laborales & 20,84 & 18,9 & 23,45 & 23,33 & 20,47 & 12,80 & 4,49 \\
\hline
\end{tabular}

FUENTE: Elaboración propia en base a Dirección General de Tributos. S.G. Política Tributaria, 2012.

La recaudación de Cooperativas por Impuesto sobre Sociedades ha pasado de 36.595,60 miles de euros en 1999 a 18.297,84 miles de euros en 2009, lo que supone una disminución en términos absolutos del 50\%. Alcanzó su máximo en el año 2006.

El Beneficio antes de Impuestos (BAI) ha pasado de 481.917,79 miles de euros en 1999 a 498.215,33 miles de euros en 2009.

El número de Cooperativas ha pasado de 25.041 en 1999 a 27.465 en 2009.

\section{Tabla 11. Ahorro Fiscal de las Cooperativas en el Impuesto sobre Sociedades, 2003-2009. Milles de euros y porcentaje}

\begin{tabular}{|l|ccccc|}
\hline AÑO & $\begin{array}{c}\text { Recaudación } \\
\text { Cooperativas }\end{array}$ & $\begin{array}{c}\text { TIE } \\
\text { Cooperativas }\end{array}$ & $\begin{array}{c}\text { TIE Agregado } \\
\text { empresas ESP }\end{array}$ & $\begin{array}{c}\text { Diferencia TIE } \\
\text { a favor Cooperativas }\end{array}$ & $\begin{array}{c}\text { Ahorro fiscal } \\
\text { cooperativas }\end{array}$ \\
\hline 2003 & $52.289,23$ & $7,05 \%$ & $24,30 \%$ & $17,25 \%$ & $127.941,73$ \\
2004 & $54.068,41$ & $8,78 \%$ & $25,00 \%$ & $16,22 \%$ & $99.884,92$ \\
2005 & $73.780,77$ & $8,62 \%$ & $24,50 \%$ & $15,88 \%$ & $135.920,95$ \\
2006 & $74.942,77$ & $7,85 \%$ & $23,30 \%$ & $15,45 \%$ & $147.498,83$ \\
2007 & $33.718,89$ & $3,48 \%$ & $19,90 \%$ & $16,42 \%$ & $159.098,90$ \\
2008 & $6.269,58$ & $0,80 \%$ & $18,00 \%$ & $17,20 \%$ & $134.795,97$ \\
2009 & $18.297,84$ & $3,67 \%$ & $16,70 \%$ & $13,03 \%$ & $64.964,81$ \\
\hline SUMA & $313.367,49$ & $5.75 \%$ & $21.67 \%$ & $15.92 \%$ & $870.106,12$ \\
\hline & & PROMEDIO & PROMEDIO & PROMEDIO & 124.300 .87 \\
\hline
\end{tabular}

FUENTE: Elaboración propia en base a Dirección General de Tributos. S.G. Política Tributaria, 2012. 


\section{EL AHORRO FISCAL DE LAS COOPERATIVAS EN EL IMPUESTO SOBRE SOCIEDADES EN ESPAÑA}

Para el año 2006, el ahorro fiscal de las cooperativas ascendió a 147.498,83 miles de euros, resultado de comparar el tipo Impositivo efectivo de la Cooperativas 7,85\% frente al $23,30 \%$ de las empresas españolas en general para ese año 2006, siendo la diferencia de $15,45 \%$ a favor de las Cooperativas.

\section{7.- Cooperativas con base imponible positiva en el Impuesto sobre Sociedades}

Una cuestión importante es el número de empresas, en este caso Cooperativas, que tributan porque presentan Base Imponible positiva. La tabla 12 ofrece estos datos.

\section{Tabla 12. Cooperativas con Base Imponible positiva}

\begin{tabular}{|lccccc|}
\hline & 2006 & 2007 & 2008 & 2009 & MEDIA \\
\hline $\mathrm{N}^{\circ}$ EMP. & 32.935 & 32.847 & 30.746 & 29.924 & $31.613,00$ \\
$\mathrm{~N}^{\circ}$ EMP. $\mathrm{Bl}>0$ & 15.966 & 15.643 & 12.200 & 11.123 & $13.733,00$ \\
$\%$ N $\mathrm{N}^{\circ}$ EMP. $\mathrm{Bl}>0$ & $48,48 \%$ & $47,62 \%$ & $39,68 \%$ & $37,17 \%$ & $43,24 \%$ \\
\hline
\end{tabular}

FUENTE: Elaboración propia en base a la información de la Agencia Estatal de la Administración Tributaria, 2012.

En la Tabla 12 se constata que en el año 2006 habían 32.935 Cooperativas activas, según los datos de Hacienda, siendo 15.966 Cooperativas las que presentaban Base Imponible positiva, lo que supone el $48,48 \%$ del total de Cooperativas con BI positiva y $51,52 \%$ con Base Imponible menor que cero.

Como puede apreciarse, el número de Cooperativas que tienen base Imponible positiva alcanzó su máximo en el año 2006, con el 48,48\%, descendiendo hasta el $37,17 \%$ en el año 2009, con el impacto de la crisis.

En conclusión, se constata que menos de la mitad de las cooperativas, en un rango que ha oscilado entre un $48 \%$ y un $37 \%$, se benefician del tratamiento fiscal especial de las cooperativas. Y éstas cooperativas son las que se benefician del ahorro fiscal estimado en el apartado anterior. 


\section{8.- Tipo Impositivo Efectivo del Impuesto sobre Sociedades por Com. Autónomas. Diferencia entre Cooperativas y Sociedades Laborales}

En este apartado, se ordenan por diferencia de la TIE entre S. Cooperativas y S. Limitadas y se hallan aquellas regiones en las que la diferencia de trato fiscal ha favorecido en mayor grado a las Cooperativas frente a las pequeñas empresas. Así, La Rioja, Madrid, Cantabria, Cataluña y CastillaLa Mancha son las más favorecidas en porcentaje. Al contrario, las menos favorecidas son Baleares, R. Murcia, Canarias, País Vasco y Galicia.

\section{Tabla 13. Diferencias regionales tributarias ordenadas por la diferencia entre la Tasa Impositiva Efectiva de las Cooperativas respecto a las Sociedades Laborales, 1999- 2009. En miles de euros y porcentaje}

\begin{tabular}{|c|c|c|c|c|c|c|c|}
\hline $\begin{array}{l}\text { MEDIA } \\
1999-2009\end{array}$ & $\begin{array}{r}\text { Recaudación } \\
\text { Economía } \\
\text { Social }\end{array}$ & $\begin{array}{l}\text { Recaudación } \\
\text { Cooperativas }\end{array}$ & $\begin{array}{r}\text { Recaudación } \\
\text { Sociedades } \\
\text { laborales }\end{array}$ & $\begin{array}{r}\text { TIE } \\
\text { Cooperativas }\end{array}$ & $\begin{array}{r}\text { TIE } \\
\text { Sociedades } \\
\text { Laborales }\end{array}$ & Diferencia & $\begin{array}{r}\text { Ventaja } \\
\text { fiscal }\end{array}$ \\
\hline LA RIOJA & 889,12 & 311,44 & 577,69 & $7,90 \%$ & $23,32 \%$ & $15,41 \%$ & 596 \\
\hline MADRID & $18.859,56$ & $3.328,52$ & $15.531,05$ & $7,36 \%$ & $22,68 \%$ & $15,32 \%$ & 7.063 \\
\hline CANTABRIA & $1.713,65$ & 322,57 & $1.391,08$ & $11,59 \%$ & $26,67 \%$ & $15,08 \%$ & 464 \\
\hline CATALUÑA & $23.213,30$ & $9.196,49$ & $14.016,81$ & $6,67 \%$ & $21,07 \%$ & $14,40 \%$ & 23.857 \\
\hline CAST.MANCHA & $8.207,21$ & $1.836,28$ & $6.370,94$ & $6,67 \%$ & $19,75 \%$ & $13,08 \%$ & 3.603 \\
\hline C.VALENCIANA & $11.128,94$ & $5.170,54$ & $5.958,40$ & $7,39 \%$ & $18,50 \%$ & $11,11 \%$ & 7.421 \\
\hline CEUTA Y MELILLA & 81,20 & 9,85 & 71,35 & $1,34 \%$ & $11,87 \%$ & $10,54 \%$ & 166 \\
\hline EXTREMADURA & $2.763,72$ & $1.479,72$ & $1.284,00$ & $8,78 \%$ & $19,05 \%$ & $10,27 \%$ & 1.612 \\
\hline ARAGÓN & $4.224,20$ & $1.590,88$ & $2.633,32$ & $8,10 \%$ & $18,13 \%$ & $10,02 \%$ & 1.868 \\
\hline CAST.y LEÓN & $6.871,64$ & $4.378,56$ & $2.493,07$ & $8,22 \%$ & $18,04 \%$ & $9,82 \%$ & 5.002 \\
\hline NAVARRA & $2.611,27$ & 353,68 & $2.257,59$ & $2,79 \%$ & $12,44 \%$ & $9,65 \%$ & 1.016 \\
\hline ASTURIAS & $2.494,13$ & 768,57 & $1.725,55$ & $11,78 \%$ & $21,34 \%$ & $9,56 \%$ & 639 \\
\hline ANDALUCIA & $16.848,41$ & $8.661,33$ & $8.187,08$ & $7,65 \%$ & $14,76 \%$ & $7,11 \%$ & 8.296 \\
\hline GALICIA & $4.307,74$ & $2.397,13$ & $1.910,61$ & $8,98 \%$ & $15,52 \%$ & $6,54 \%$ & 1.589 \\
\hline PAIS VASCO & $48.018,87$ & $41.107,76$ & $6.911,11$ & $12,92 \%$ & $19,20 \%$ & $6,28 \%$ & 19.981 \\
\hline CANARIAS & $1.466,78$ & 359,17 & $1.107,61$ & $4,71 \%$ & $10,88 \%$ & $6,18 \%$ & 478 \\
\hline R.MURCIA & $8.154,07$ & $4.555,36$ & $3.598,70$ & $15,14 \%$ & $17,25 \%$ & $2,11 \%$ & 4.299 \\
\hline BALEARES & $1.994,07$ & 530,26 & $1.463,81$ & $14,38 \%$ & $13,19 \%$ & $-1,19 \%$ & -77 \\
\hline
\end{tabular}

FUENTE: Elaboración propia en base a la información de la Agencia Estatal de la Administración Tributaria, 2012. 
Tabla 14. Resumen de las diferencias regionales de las ventajas fiscales ordenadas por magnitud de la ventaja fiscal. Media 1999-2009. En miles de euros y porcentaje

\begin{tabular}{|l|r|r|r|r|r|r|r|r|}
\hline MEDIA & $\begin{array}{r}\text { Recaudación } \\
\text { 1999-2009 }\end{array}$ & $\begin{array}{r}\text { Recaud. } \\
\text { Sociala }\end{array}$ & $\begin{array}{r}\text { Recaudación } \\
\text { Cociedades }\end{array}$ & $\begin{array}{r}\text { TIE } \\
\text { Coops } \\
\text { laborales }\end{array}$ & $\begin{array}{r}\text { TIE } \\
\text { Socied. } \\
\text { Laborales }\end{array}$ & Diferencia & $\begin{array}{r}\text { Ventaja } \\
\text { fiscal }\end{array}$ & $\%$ \\
\hline CATALUÑA & $23.213,30$ & $9.196,49$ & $14.016,81$ & $6,67 \%$ & $21,07 \%$ & $14,40 \%$ & 23.857 & $27,15 \%$ \\
PAIS VASCO & $48.018,87$ & $41.107,76$ & $6.911,11$ & $12,92 \%$ & $19,20 \%$ & $6,28 \%$ & 19.981 & $22,74 \%$ \\
ANDALUCIA & $16.848,41$ & $8.661,33$ & $8.187,08$ & $7,65 \%$ & $14,76 \%$ & $7,11 \%$ & 8.296 & $9,44 \%$ \\
C.VALENCIANA & $11.128,94$ & $5.170,54$ & $5.958,40$ & $7,39 \%$ & $18,50 \%$ & $11,11 \%$ & 7.421 & $8,45 \%$ \\
MADRID & $18.859,56$ & $3.328,52$ & $15.531,05$ & $7,36 \%$ & $22,68 \%$ & $15,32 \%$ & 7.063 & $8,04 \%$ \\
CAST.y LEÓN & $6.871,64$ & $4.378,56$ & $2.493,07$ & $8,22 \%$ & $18,04 \%$ & $9,82 \%$ & 5.002 & $5,69 \%$ \\
R.MURCIA & $8.154,07$ & $4.555,36$ & $3.598,70$ & $15,14 \%$ & $17,25 \%$ & $2,11 \%$ & 4.299 & $4,89 \%$ \\
CAST.MANCHA & $8.207,21$ & $1.836,28$ & $6.370,94$ & $6,67 \%$ & $19,75 \%$ & $13,08 \%$ & 3.603 & $4,10 \%$ \\
ARAGÓN & $4.224,20$ & $1.590,88$ & $2.633,32$ & $8,10 \%$ & $18,13 \%$ & $10,02 \%$ & 1.868 & $2,13 \%$ \\
EXTREMADURA & $2.763,72$ & $1.479,72$ & $1.284,00$ & $8,78 \%$ & $19,05 \%$ & $10,27 \%$ & 1.612 & $1,83 \%$ \\
GALICIA & $4.307,74$ & $2.397,13$ & $1.910,61$ & $8,98 \%$ & $15,52 \%$ & $6,54 \%$ & 1.589 & $1,81 \%$ \\
NAVARRA & $2.611,27$ & 353,68 & $2.257,59$ & $2,79 \%$ & $12,44 \%$ & $9,65 \%$ & 1.016 & $1,16 \%$ \\
ASTURIAS & $2.494,13$ & 768,57 & $1.725,55$ & $11,78 \%$ & $21,34 \%$ & $9,56 \%$ & 639 & $0,73 \%$ \\
LA RIOJA & 889,12 & 311,44 & 577,69 & $7,90 \%$ & $23,32 \%$ & $15,41 \%$ & 596 & $0,68 \%$ \\
CANARIAS & $1.466,78$ & 359,17 & $1.107,61$ & $4,71 \%$ & $10,88 \%$ & $6,18 \%$ & 478 & $0,54 \%$ \\
CANTABRIA & $1.713,65$ & 322,57 & $1.391,08$ & $11,59 \%$ & $26,67 \%$ & $15,08 \%$ & 464 & $0,53 \%$ \\
CEUTA Y MELILLA & 81,20 & 9,85 & 71,35 & $1,34 \%$ & $11,87 \%$ & $10,54 \%$ & 166 & $0,19 \%$ \\
BALEARES & $1.994,07$ & 530,26 & $1.463,81$ & $14,38 \%$ & $13,19 \%$ & $-1,19 \%$ & -77 & $-0,09 \%$ \\
\hline & & & & & & & 87.879 & $100 \%$ \\
\hline
\end{tabular}

FUENTE: Elaboración propia en base a la información de la Agencia Estatal de la Administración Tributaria, 2012. 


\section{9.- Conclusiones}

La presión fiscal en España respecto al PIB es del 35\% para la década 1998-2008, siendo la presión fiscal del Impuesto sobre Sociedades del 3,37\% de ese 35\%.

Los Tributos recaudados en la década 1999-2009 suponen una media anual de recaudación de 108.012,84 millones de euros, es decir, lo recaudado por Impuesto sobre Sociedades supone un $23,60 \%$. La presión fiscal, entendida como la suma de Cotizaciones Sociales más Tributos sobre el PIB, durante el periodo 1998-2008, supuso como media en España un 35\% del PIB. Siguiendo un orden de importancia las Cotizaciones Sociales suponen un 12,21\% del total; en segundo lugar la recaudación del IRPF, que supone el 6,89\% del total; en tercer lugar el IVA, que supone el 5,97\% del total. Otros impuestos Indirectos representan el 3,39\% del total; en quinto lugar, el Impuesto sobre Sociedades ocuparía el sexto lugar en recaudación, con el 3,37\% del total de la presión fiscal que hemos dicho que era el 35\% del PIB. Seguirían Impuestos Especiales, con el 2,45\%, y Otros impuestos directos, con el $0,81 \%$. A nivel sólo de ingresos tributarios, tomando como referencia el año 2006, de una recaudación total de 130.126,50 millones de euros, la recaudación por Impuesto sobre Sociedades ascendió a 37.207,60 millones de euros, lo que supone un porcentaje del $28,59 \%$ del total recaudado. La evolución ha sido desde el 15,37\% en 1999 al 24,14\% en 2009.

La recaudación por Impuesto sobre Sociedades de la Economía Social supone un 0,43\% del total, la recaudación por IS de las Cooperativas supone un $0,18 \%$ del total de lo recaudado por Impuesto sobre Sociedades promedio de la década 1999-2009.

El peso de la recaudación por Impuesto sobre Sociedades de la Economía Social en general en España para el periodo 1999-2009 es el siguiente: Recaudación por Impuesto sobre Sociedades, promedio de 25.495,45 millones de euros (100\%), recaudación por IS Cooperativas, 42,81 millones de euros $(0,18 \%)$, recaudación ISS Laborales 68,02 millones de euros $(0,25 \%)$ y Economía Social (suma de Coop + SLab) 110,83 millones de euros (0,43\%).

La ventaja fiscal o ahorro fiscal de las Cooperativas frente a las Sociedades Laborales, que en el estudio se han equiparado a las empresas capitalistas, sólo a efectos de tributación, asciende a 1.002,28 millones de euros, sumando todos los años desde 1999 hasta 2009, es decir, una media de 91,11 millones de euros anuales.

Calculado con una diferencia de Tasa Impositiva Efectiva (TIE) de las Cooperativas, con una media de 6,34\% TIE, y las Sociedades Laborales con una media de TIE del 19,17\%, lo que supone una diferencia en porcentaje de $12,83 \%$, de ventaja fiscal de las Cooperativas frente a las Sociedades Laborales. 


\section{EL AHORRO FISCAL DE LAS COOPERATIVAS EN EL IMPUESTO SOBRE SOCIEDADES EN ESPAÑA}

Esa ventaja fiscal si se compara Cooperativas con el Tipo Impositivo Efectivo Total Agregado (TIETA) en España 2003-2009, respecto al Impuesto sobre Sociedades, todavía nos daría magnitudes superiores con una ventaja fiscal de las Cooperativas sobre el Agregado Total de casi $16 \%$ de ventaja fiscal cercana a los 870,10 millones de euros pero para tan sólo 7 años, no 11 como hemos visto en la conclusión anterior (supone 124,30 millones de euros al año).

Al realizar una extrapolación a once años los 124,30 millones de euros anuales, saldría una magnitud de Ventaja Fiscal/Ahorro Fiscal de las Cooperativas comparado con el Agregado total 1.367,30 millones de euros. Es decir, la ventaja fiscal de las Cooperativas en España se puede estimar entre 1.002,28 y 1.367,30 millones de euros desde 1999 hasta 2009 (es decir unos 107,70 millones de euros al año).

Los beneficios de las Cooperativas provienen de actividades ordinarias en un $50,56 \%$ frente a actividades extraordinarias del $49,44 \%$.

La proporción entre beneficios ordinarios frente a beneficios extraordinarios de las Cooperativas en España durante el periodo 1999-2007 está en un 50,56\% Resultado de Actividades ordinarias (RAO), frente a un 49.44\% Resultado de Actividades Extraordinarias (RAE) de media, en base a esto se puede decir que está igualada, esa situación cambia para las Sociedades Laborales cuyos resultados son $65,68 \%$ RAO, 34,22\% RAE.

Las Cooperativas en su conjunto tienen un volumen de beneficios que representa el $68,55 \%$ y su volumen de pérdidas es de $31,45 \%$ restante.

La Proporción de volumen de beneficios frente a volumen de pérdidas es para las Cooperativas en el periodo 1999-2009 de una media de 68,55\% Volumen de Beneficios, 31,45\% Volumen de pérdidas. Las Sociedades Laborales arrojan el resultado de 57,36\% Volumen de Beneficios, $42,64 \%$ Volumen de Pérdidas.

El número de Cooperativas con Base Imponible Positiva, es decir con beneficios son como media para el periodo 2006-2009 de 13.733 sociedades Cooperativas que suponen un $43,24 \%$ del total que son 31.613 sociedades Cooperativas.

Es decir, el $43,24 \%$, es decir, 13.733 Cooperativas obtienen el $68,55 \%$ de los beneficios. El $56,76 \%$, esto es, las 17.880 Cooperativas restantes absorben el $31,45 \%$ de las pérdidas.

Supondría una subvención de 107,70 millones de euros al año al repartir entre 13.733 empresas lo que da una subvención por ventaja fisca/ ahorro fiscal de 7.842,42 euros por empresa. Esta conclusión es muy importante a la hora de analizar el éxito de las Cooperativas y su política de beneficios. Sobre todo en lo que respecta a Cooperativas que tiendan a beneficio cero o pérdidas. 
La Comunidad Autónoma que tiene más ventaja fiscal / ahorro fiscal en porcentaje es La Rioja con un ahorro fiscal del 15,41\% i.e. 0,59 millones de euros al año de media, en segundo lugar Comunidad de Madrid ahorro fiscal del 15,32\% que le supone 7,063 millones al año, en tercer lugar Cantabria ahorra el $15,08 \%$ que le supone 0,464 millones de euros, Cataluña en cuarto lugar con un ahorro del $14,40 \%$ que le supone 23,85 millones de euros al año, en quinto lugar con un ahorro del $13,08 \%$ Castilla-La Mancha ahorro fiscal del 13,08\% le suponen 3,60 millones al año.

Las diferencias regionales dentro de España, como media durante el periodo 1999-2009, por diferencia de Tipo Impositivo Efectivo entre Cooperativas y Sociedades Laborales oscila desde 15,41\% de media en La Rioja hasta el -1,19 en Baleares. Es decir, las mayores diferencias de TIE se darían en La Rioja, Madrid, Cantabria, Cataluña y Castilla-La Mancha. Las menores diferencias de TIE entre Cooperativas y Sociedades Laborales las tenemos en Baleares, Murcia, Canarias, País Vasco y Galicia.

Se puede extraer qué Comunidad Autónoma paga más, atendiendo al Tipo Efectivo Impositivo: en primer lugar la Región de Murcia, con un TIE en sus Cooperativas del 15,14\% y una recaudación de 4,55 millones de euros de media, 1999-2009; en segundo lugar Baleares, con un TIE del 14,38\% y 0,53 millones de euros; en tercer lugar País Vasco, con una TIE del $12,20 \%$ y una recaudación meda de 41,10 millones de euros; el cuarto lugar es para Asturias, con una TIE del $11,78 \%$ y una recaudación meda de 0,76 millones de euros.

Las diferencias regionales ordenadas por importe, en miles de euros de la ventaja fiscal/ahorro fiscal, oscilan entre los 23.857,76 miles de euros de media para Cataluña en el periodo 1999-2009, hasta Baleares con un resultado negativo de 77,66 miles de euros. Es decir, las regiones que más ventaja fiscal han tenido, en miles de euros, han sido Cataluña, País Vasco, Andalucía, Comunidad Valenciana y Madrid. Las regiones que menos importe han obtenido como ventaja fiscal han sido Baleares, Ceuta y Melilla, Cantabria, Canarias y La Rioja.

¿Qué comunidades han tenido mayor ahorro fiscal / ventaja fiscal? Atendiendo al importe en millones de euros, en primer lugar Cataluña, con un ahorro de 23,85 millones de euros y ventaja del $14,40 \%$; en segundo lugar País Vasco, 19,98 millones de euros de ahorro y un 6,28\% de ventaja; en tercer lugar Andalucía, con 8,92 millones de euros y una ventaja del 7,11\%; en cuarto lugar la Comunidad Valenciana, con 7,063 millones de euros de ahorro anual de media y una ventaja del $11,11 \%$ sobre las Sociedades laborales, y en quinto lugar la Comunidad de Madrid, con 7,063 millones de ahorro medio anual y una ventaja del $15,32 \%$.

¿Qué comunidades han tenido menor ahorro fiscal / ventaja fiscal? Atendiendo al importe en millones de euros, en primer lugar Baleares, con un desahorro de 0,07 millones de euros y desventaja del 1,19\%; en segundo lugar Ceuta y Melilla, 0,166 millones de euros de ahorro y un 10,54\% de ventaja; en tercer lugar Cantabria, con un ahorro de 0,464 millones de euros y una ventaja del 15,08\%; en cuarto lugar Canarias, con un ahorro desde 0,464 millones de euros y una ventaja del 6,18\%, y en quinto lugar La Rioja, con un ahorro de 0,596 millones de euros y una ventaja del $15,41 \%$. 


\section{EL AHORRO FISCAL DE LAS COOPERATIVAS EN EL IMPUESTO SOBRE SOCIEDADES EN ESPAÑA}

\section{Bibliografía}

AGENCIA TRIBUTARIA: Estadísticas tributarias. Informes estadísticos. Documento en línea $\mathrm{http} / /$ www.agenciatributaria.es/AEAT.internet/Inicio_es_ES/La_Agencia_Tributaria/Memorias_y_estadisti cas_tributarias/Estadisticas_tributarias/Estadisticas_tributarias.shtml Varias consultas [2011-2012].

ALBI, E., GONZÁLEZ-PÁRAMO, J.M. \& ZUBIRI, I. (2009): Economía Pública, Editorial Ariel, Barcelona.

ALGUACIL, M.P. (2011): "Fiscalidad de Cooperativas y Ayudas de Estado: Parámetros para una reforma", Instituto de Estudios Fiscales. Documento de trabajo 2/2011.

CALVO, R. (2005): "Entidades de Economía Social: razones de una fiscalidad específica". En: Fiscalidad de las entidades de Economía Social. Cooperativas, Mutuas, Sociedades Laborales, Fundaciones, Asociaciones de Utilidad pública, Centros Especiales de Empleo, Empresas de Inserción Social, Editorial Thomson-Civitas-Aranzadi.

CIRIEC-España: http://www.ciriec.es/ Varias consultas [2011-2012].

DOMÍNGUEZ, F., LÓPEZ, J. \& TODRIGO, F. (2005): "¿Afectan el IRPF y el Impuesto sobre Sociedades a la elección de la forma de empresa?", Hacienda Pública Española, 174, 55-86. En línea: http://www.ief.es/documentos/recursos/publicaciones/revistas/hac_pub/174_IRPF.pdf. Fecha de consulta [10.02.2015]

CHAVES, R. (2012): "Las Políticas Públicas y las Cooperativas", Ekonomiaz, 79, 169-200.

CHAVES, R. \& MONZÓN, J.L. (2008): La Economía Social en la Unión Europea, Comité Económico y Social Europeo, Bruselas.

FERNÁNDEZ, E., MARTÍNEZ, A. \& ÁLVAREZ, S. (2008): "La presión fiscal por el impuesto sobre sociedades en la Unión Europea", FUNCAS, Documento de trabajo, 407/2008. En línea http://www.elconfidencial.com/fotos/sociedades0109.pdf. Fecha de consulta [10.02.2015]

INSTITUTO UNIVERSITARIO DE ECONOMÍA SOCIAL Y COOPERATIVA (IUDESCOOP). En línea http://www.uv.es/iudescoop/ Varias consultas [2011.2012].

JULIÁ, J.F. \& SERVER, R.J. (1996): "La fiscalidad de las Cooperativas en el marco de la Unión Europea. Referencia especial al caso español", CIRIEC-España, Revista de Economía Pública, Social y Cooperativa, 23, 47-69.

MARÍN SÁNCHEZ, M.M., \& SERVER IZQUIERDO, R.J. (2004): "La fiscalidad de empresas de economía social", Mediterráneo Económico, 6, 223-237. En línea:

http://www.fundacioncajamar.es/mediterraneo/revista/ecosocial.pdf. Consulta: [15.01.2014]. 
MINISTERIO DE EMPLEO Y SEGURIDAD SOCIAL. Empleo. Economía Social. Datos estadísticos de la Economía Social. Información fiscal de las sociedades de Economía Social. En línea: http://www.empleo.gob.es/es/sec_trabajo/autonomos/economiasoc/EconomiaSocial/estadisticas/DatosFiscales/index.htm. Varias consultas [2011.2012].

MINISTERIO DE HACIENDA Y ADMINISTRACIONES PÚBLICAS. Estadísticas. Impuestos. Documento en línea: http://www.minhap.gob.es/esES/Estadistica\%20e\%20Informes/Impuestos/Paginas/Impuestos.aspx. Varias consultas [20112012].

MONSERRAT, J. (1991): "Hacia una teoría económica del Tercer Sector, una primera aproximación", Economistas, 51, 48-54.

OBSERVATORIO ESPAÑOL DE LA ECONOMÍA SOCIAL En línea: http://www.observatorioeconomiasocial.es/ Varias consultas [2011-2012].

RODRIGO RUIZ, M.A. (2010): "Consideraciones sobre el régimen fiscal de las Cooperativas. Problemas actuales y líneas de reforma", CIRIEC-España, Revista de Economía Pública, Social y Cooperativa, 69, 9-25. 